\title{
Bosutinib in chronic myeloid leukemia: patient selection and perspectives
}

This article was published in the following Dove Press journal:

Journal of Blood Medicine

\author{
Susanne Isfort $^{1,2}$ \\ Tim H Brümmendorf ${ }^{\prime}$ \\ 'Department of Hematology, \\ Oncology, Hemostaseology and Stem \\ Cell Transplantation, Medical Faculty, \\ RWTH Aachen University, Aachen, \\ Germany; ${ }^{2}$ Center for Translational \\ and Clinical Research Aachen \\ (CTC-A), Medical Faculty at the \\ RWTH Aachen University, Aachen, \\ Germany
}

\begin{abstract}
During recent years, the therapeutic landscape in chronic myeloid leukemia (CML) has changed significantly. Since the clinical introduction of tyrosine kinase inhibitors (TKIs) approximately 15 years ago, patients' concerns have shifted from reduced life expectancy toward long-term toxicities of TKI, depth of remission, and the probability of successful treatment discontinuation. Patients with newly diagnosed CML in chronic phase (at least with a Sokal score not exceeding intermediate) may now expect an almost normal life expectancy. However, even if almost $30 \%$ of all newly diagnosed chronic-phase patients might eventually be facing the prospect of a life without CML-specific treatment, based on current knowledge, most, if not all, patients would have to undergo an expected minimum of 5-8 years of TKI treatment and the majority would face a life-long exposure to the side-effects of TKIs. At present, 5 different TKIs are licensed for the treatment of CML, that is, imatinib, which is a first-generation TKI (including its generic derivatives); nilotinib, dasatinib, and bosutinib, which are second-generation TKIs; as well as ponatinib, which is a so-called third-generation TKI and is supposed to be used for patients harboring the T315I-mutation. One of the important, yet unanswered questions is the choice of the best possible TKI upfront for each individual patient. Bosutinib is currently licensed for patients with CML after failure or intolerance of at least 2 other TKIs. It can also be prescribed according to label if after failure of the first TKI therapy, another option does not seem feasible. This review focuses on the existing data on clinical efficacy, tolerability, and side effects of bosutinib treatment in CML patients with the aim to identify patient characteristics and treatment scenarios most suitable for treatment with bosutinib.
\end{abstract} Keywords: tyrosine kinase inhibitors, side effect profile, individual comorbidity profile

\section{Introduction}

Bosutinib (SKI-606, Bosulif ${ }^{\mathbb{B}}$; Pfizer, New York, NY, USA) acts as a dual inhibitor of Src and ABL kinases. Compared with other second-generation tyrosine kinase inhibitors (TKIs), it has only minimal inhibitory activity against platelet-derived growth factor receptor -A or c-KIT.,

Only low concentrations of bosutinib are required to ablate BCR-ABL phosphorylation when compared with the first-generation TKI imatinib (Glivec ${ }^{\mathbb{R}}$, Novartis, Basel, Switzerland). Bosutinib is a potent second-generation inhibitor of chronic myeloid leukemia (CML) cell proliferation in vitro and has been found to be capable of overcoming the majority of imatinib-resistant BCR-ABL mutations. ${ }^{1,3}$ A detailed review on the biochemical, pharmacokinetic, and pharmacodynamic properties of the drug is given in several publications, including reviews. ${ }^{4-6}$

Bosutinib is administered once daily and its absorption increases with food. ${ }^{4}$ It is, therefore, recommended to be taken with food, which, might be more convenient and
Correspondence: Susanne Isfort Department of Hematology, Oncology, Hemostaseology and Stem Cell Transplantation, University Hospital Aachen, Pauwelsstrasse 30, 52074

Aachen, Germany

Email sisfort@ukaachen.de 
potentially increases adherence for some patients as - compared with other TKIs - for example between Nilotinib intake and meals, an interval of at least 2 hours has to be respected. Bosutinib, though recommended, can either be taken with or without food. Bosutinib has an extensive tissue distribution and is primarily metabolized by cytochrome $\mathrm{p} 450$ isoenzyme 3A4 (CYP3A4). Preexisting renal insufficiency should be considered before treatment with bosutinib is initiated since Cortes et al recently reported that long-term bosutinib treatment may cause a modest but significant, apparently reversible decline in renal function. ${ }^{7}$ Consequently, close monitoring of patients with impaired renal function is recommended under bosutinib treatment. ${ }^{7}$

Until now, bosutinib has been licensed for patients with Philadelphia-positive CML in chronic phase (CP), accelerated phase (AP), and blast crisis (BC) having been treated with at least 1 prior TKI and in which using imatinib, dasatinib (Sprycel ${ }^{\mathbb{}}$, Bristol-Myers Squibb, Otsuka America Pharmaceuticals Inc., San Francisco, CA, USA), or nilotinib (Tasigna $^{\circledR}$, Novartis, Basel, Switzerland) does not seem to be an appropriate choice. This license was based mainly on a Phase I/II trial, including patients in $\mathrm{CP}, \mathrm{AP}$, and $\mathrm{BC}$ (NCT00261846). ${ }^{8,9}$

\section{Bosutinib in advanced clinical stages of CML}

In advanced stage CML, that is, $\mathrm{AP}$ or $\mathrm{BC}$, bosutinib seems to provide rather stable long-term efficacy in patients who show an initial response to treatment. Gambacorti-Passerini et al reported the long-term efficacy and safety of the drug in advanced leukemia patients after prior treatment failure in a Phase I/II trial (NCT00261846). ${ }^{10}$ Seventy-nine patients with AP, 64 in BC, and 24 with Philadelphia-chromosome positive $(\mathrm{Ph}+)$ acute lymphocytic leukemia (ALL) had been treated with bosutinib in this trial. Most of these patients were highly pretreated, 9\% even with 3 lines of TKI treatment. Longterm survival is still limited in this highly selected patient cohort; however, especially within the group of patients in AP, durable responses were achieved. With a median follow-up of 28.4 months (range 0.3-88.6), median overall survival (OS) in this cohort was not reached, 30 patients died, 11 among them within 30 days of their last bosutinib dose. Even among the patients with $\mathrm{Ph}+\mathrm{ALL}$, few long-lasting responses were noted, for example, 1 patient maintained his initial complete hematologic response (CHR) for $>304.3$ weeks and a complete cytogenetic response (CCyR) for 327 weeks. Overall, and based on response rates in similar but obviously not identically laid out non-randomized trials, bosutinib seems to be similarly effective as the other secondgeneration TKIs approved in this situation. With about $50 \%$ of the AP responders and $25 \%$ of $\mathrm{BC}$ patients maintaining their response after 4- and 1-year treatment, respectively, bosutinib represents an additional treatment option not only for patients with a non-curative approach but also as a bridging therapy to allogeneic stem cell transplantation. Atilla et al reported about a patient with secondary lymphoid blast phase with suspected central nervous system involvement transformed from a CPCML pretreated with imatinib, dasatinib, and nilotinib. ${ }^{11}$ In this case, treatment with bosutinib resulted in a durable hematologic response and was even able to maintain central nervous system response when combined with intrathecal chemotherapy.

In the framework of the same study, 3 different quality of life (QoL) assessment tools were used. ${ }^{12}$ During treatment, almost all patients experienced significant increases in their QoL under bosutinib treatment independent of whether they were in AP or blastic phase of CML.

\section{Bosutinib in second and later lines in $\mathrm{CP}$ patients}

Bosutinib has been tested in the second-, third-, and fourthline setting in a Phase I/II trial (NCT00261846).

In the context of this clinical trial, 288 patients were treated with bosutinib as second-line therapy after imatinib failure (the distribution of patients in the different strata of the trial is shown in Figure 1).

The data from the 2-year follow-up of this study was published in 2014 showing that $85 \%$ achieved/maintained CHR, 59\% achieved/maintained major cytogenetic response (MCyR, including 48\% with CCyR), and 35\% achieved major molecular response (MMR). ${ }^{9} \mathrm{OS}$ and progression-free survival (PFS) rates after 2 years of treatment were $91 \%$ and $81 \%$, respectively. In a more mature analysis of this trial, Brümmendorf et al focused on identifying factors that might have an influence on long-term outcome. ${ }^{16} \mathrm{BCR}-\mathrm{ABL} 1$ mutations could be identified as significant predictor of decreased OS with a hazard ratio (HR) of 3.35 .

Results have already been reported in 2012 and have recently been updated with long-term outcomes. ${ }^{8,13,14}$ Median follow-up of the 119 patients included in the analysis was 32.7 months. MCyR rate after 4 years was $40 \%$, the probability of maintaining MCyR was $69 \%$. OS was $78 \%$ after 4 years. The incidence of AP transformation was only $4 \%, 13 \%$ of 57 patients developed an additional/new mutation leading to discontinuation of TKI in 12 of the 13 cases mostly because of lack of efficacy. 


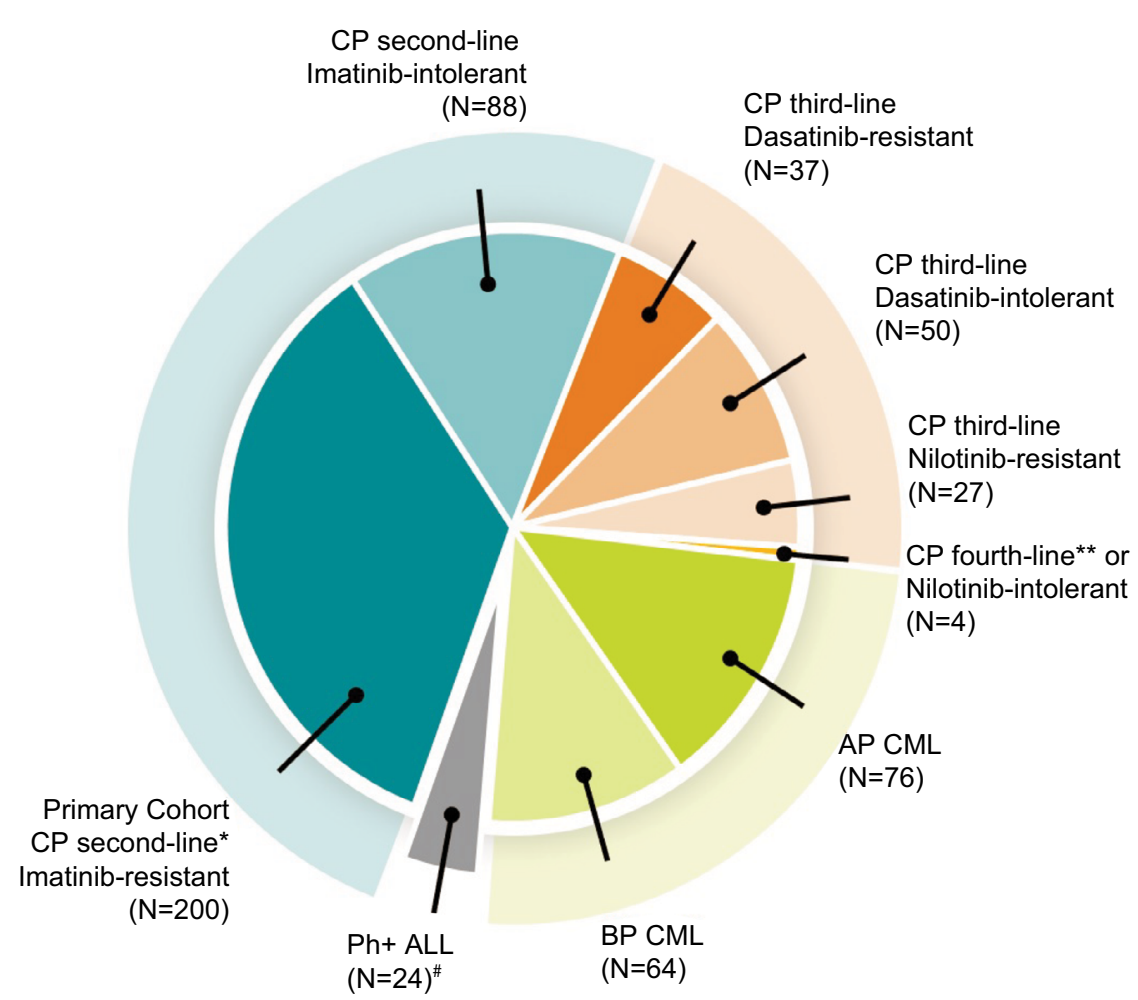

Figure I Study 200: Patient cohorts $(n=570)$ in second+ line.

Notes: Data from Cortes et al..$^{15}$ indicates bosutinib is not indicated for Ph+ ALL; *indicates primary efficacy endpoint of the study was the MCyR rate at week 24 in patients with imatinib-resistant CP CML previously treated with only one prior TKI (imatinib); **indicates three chronic phase CML patients in fourth-line, and one nilotinib intolerant patient in third-line treatment.

Abbreviations: ALL, acute lymphocytic leukemia; AP, accelerated phase; BP, blastic phase; CML, chronic myeloid leukemia; CP, chronic phase; MCyR, major cytogenetic response.

Regarding QoL assessments in this study, only a small health-related QoL impairment was demonstrated at baseline. During the 96 weeks on bosutinib therapy, patients reported statistically significant and/or clinically meaningful improvements on several Functional Assessment of Cancer Therapy-Leukemia scales. ${ }^{17}$ Based on the aforementioned study, bosutinib was approved in 2012 in Europe and the USA. After approval, another Phase IV-trial was initiated in Europe testing bosutinib in label and will soon provide more safety and efficacy data out of a real-life setting (NCT02228382). This trial also included patients in fourth line of therapy. Data on bosutinib treatment in fourth-line treatment is rare. In Spain, a retrospective analysis of 30 CPCML patients after failure of 3 different TKIs (imatinib + nilotinib + dasatinib) was performed. ${ }^{18}$ At the time of data cutoff, median follow-up after bosutinib initiation was 11.5 months (range: $2.0-46.0$ months) and median duration of treatment across all cohorts was 9.3 months (range: 0.6-23.4 months). Median time for achievement of best response was short (3.7 months [range: $0.2-15.6$ months]). Ninty percent (27/30) of patients achieved or maintained CHR. The probability to either maintain or improve their CCyR response was 56.6\% (17/30) and 11 patients $(36.7 \%)$ achieved or maintained their baseline
MMR. In patients with responses of less than CCyR at baseline, the probabilities of obtaining CCyR, MMR, and MR4.5 were $13 \%, 11 \%$, and $14 \%$, respectively. PFS was $73 \%$ at 20 months.

\section{Bosutinib in first-line treatment}

Bosutinib was primarily tested in the first-line setting at a daily dose of $500 \mathrm{mg}$ vs imatinib standard dose of $400 \mathrm{mg}$ in the so-called BELA trial. Unfortunately, this study missed its primary endpoint because the CCyR-rate at 12 months with bosutinib was not significantly different from the CCyR rate of the imatinib-treated patients in the control arm (NCT00574873). However, all clinically meaningful secondary endpoints were significantly different in favor of bosutinib in this study. ${ }^{19-21}$ Importantly, no excess cardiovascular toxicity had been reported at this dosage compared with imatinib given at the $400 \mathrm{mg}$ standard dose.

After thorough analysis of the BELA study data, including efficacy and safety of individual dose levels of bosutinib, as well as based on the experience gained from other secondgeneration TKIs suggesting that first-line treatment requires lower doses of TKI compared with second- and later-line treatments, a reduced daily dose of $400 \mathrm{mg}$ bosutinib was investigated again in first-line setting compared with imatinib 
standard dose within the so-called BFORE trial, which closed recruitment in 2015 (NCT02130557). At the end of 2016, it was announced that this study met the primary endpoint meaning that bosutinib was superior to imatinib in first-line treatment regarding the MMR rate (and also again, regarding CCyR rate) at 12 months of treatment, these data were recently confirmed with 18 months follow-up..$^{23,24}$

In the BFORE trial, 536 patients with newly diagnosed CP-CML were randomized 1:1 to receive $400 \mathrm{mg}$ of bosutinib once daily $(n=268)$ or imatinib $(n=268) .{ }^{23}$ The median dose intensity for bosutinib was 392 and $400 \mathrm{mg} / \mathrm{d}$ for imatinib. The MMR rate at 12 months was significantly higher with bosutinib vs imatinib (47.2\% vs $36.9 \% ; P=0.02)$. Furthermore, CCyR rate by 12 months $(77.2 \%$ vs $66.4 \% ; P=0.0075)$ was also increased significantly. Time to response was much shorter in bosutinib-treated patients. Only few patients in both arms ( $1.6 \%$ of bosutinib and $2.5 \%$ of imatinib patients) progressed to accelerated/blast phase within the 12 months follow-up period. Whereas more patients discontinued treatment due to drug-related toxicity $(12.7 \%$ for bosutinib and $8.7 \%$ for imatinib), this was outweighed in favor of bosutinib by a lower rate of discontinuations for lack of efficacy or related reasons. The safety profile of bosutinib in this trial was overall consistent with former trials; however, the grade $>3$ diarrhea rate was reduced to $7.8 \%$ compared with $11 \%$ in the BELA trial potentially as a result of the initial dose reduction as well as improved patient management under treatment due to increased alertness of the treating physicians for this toxicity. ${ }^{19,23}$

\section{Safety of bosutinib}

The non-hematological safety profile of bosutinib is different compared with the other approved TKIs in some concerns. Side-effect profiles are increasingly being used for selection of TKIs in first- and later-line treatments given that efficacy is overall not dramatically different for all approved second-generation TKIs and therefore adherence as well as long-term tolerability become increasingly important. Table 1 is adapted from a publication by Gambacorti-Passerini et al and (despite missing direct comparisons available for the second-generation TKIs) tries to weigh the predominant typical side-effect profiles of currently approved TKIs for CML treatment on the basis of a non-quantitative cross-trial comparison. $^{25}$

Under bosutinib treatment, $>80 \%$ of patients experience gastrointestinal adverse events (AEs), in particular episodes of nausea, vomiting, and especially diarrhea. Most of these side effects occur during the first few weeks of treatment, are self-limiting, and normally of low grade. Several publications give advice regarding prophylactic treatment in order to avoid significant GI toxicity. In the later trials, patients have been equipped with extensive instructions in order to avoid severe or long-lasting episodes of diarrhea, for example, using loperamid treatment and in individual cases, even prophylaxis. Episodes of higher grade diarrhea were much less common and reported to occur in the order of $10^{+\%} \%$ in several studies; however, treatment discontinuation due to diarrhea was a rare event. ${ }^{26}$

As most of the other TKIs, bosutinib can cause skin rashes in about $30 \%$ of patients. ${ }^{26}$ However, skin rashes are usually reported to be short-lasting and generally well manageable. In the publication by Ault et al, the authors give advice on what behavior to avoid (long baths, tight clothing) and which actions to take (eg. application of topical antiseptics or topical antibiotics).

Transaminase elevations and lipase increases were also reported significantly more frequently under bosutinib than imatinib treatment and are the most important reason for discontinuation of bosutinib. However, these toxicities once monitored closely can mostly be handled successfully by treatment interruptions and/or dose reductions and, eventually, most patients can continue with the drug upon reexposure.

As for all other TKIs used in CML treatment, bosutinib side effects in the hematopoietic system are, at least partly, thought to reflect the efficacy of the drug. However, while the rate of neutropenias is in favor of bosutinib, thrombocytopenia was the most common cause for treatment discontinuation in the first-line BELA trial. ${ }^{19}$ This is rather

Table I Most frequent side effects of all approved TKI

\begin{tabular}{|c|c|c|c|c|c|}
\hline & Imatinib & Nilotinib & Dasatinib & Bosutinib & Ponatinib \\
\hline Hematologic & Neutropenia & & $\begin{array}{l}\text { Neutropenia, } \\
\text { thrombocytopenia }\end{array}$ & & \\
\hline Lab values & Phosphate & $\begin{array}{l}\text { Lipase, ALT, AST, } \\
\text { blood glucose }\end{array}$ & $\begin{array}{l}\text { Changes in coagulation } \\
\text { parameters }\end{array}$ & ALT, AST, Lipase & $\begin{array}{l}\text { Lipase, blood glucose, } \\
\text { cholesterol, triglyceride }\end{array}$ \\
\hline Non-lab AEs & $\begin{array}{l}\text { Muscle cramps, } \\
\text { edema }\end{array}$ & $\begin{array}{l}\text { Rash, PAOD, other } \\
\text { vascular events }\end{array}$ & $\begin{array}{l}\text { Pleural effusion, Pulmonal } \\
\text { hypertension }\end{array}$ & $\begin{array}{l}\text { Nausea, vomiting, } \\
\text { diarrhea }\end{array}$ & $\begin{array}{l}\text { Rash, PAOD, arterial and } \\
\text { venous events, arterial } \\
\text { hypertension }\end{array}$ \\
\hline
\end{tabular}

Abbreviation: AEs, adverse events; ALT, alanine transaminase; AST, aspartate transaminase; PAOD, peripheral arterial vascular occlusive disease; TKI, tyrosine kinase inhibitors. 
surprising, since lacking activity against c-kit and/or PDGF-R was initially hypothesized to result in a favorable hematologic side-effect profile of the drug. ${ }^{27,28}$ Overall, and in line with other second-generation TKIs and imatinib, cytopenias can usually be managed well with transient treatment interruptions and dose modifications.

Cross-intolerance of bosutinib seems to be rare. In the retrospective analysis published by Garcia-Gutierrez et al, in heavily pretreated patients exposed to bosutinib as fourth-line treatment, only few patients re-developed side effects previously experienced with other TKIs. ${ }^{18}$ Fifteen patients who suffered from pleural effusions under dasatinib treatment did not experience new episodes of pleural effusions. No vascular event occurred among the 10 patients that had experienced vascular events under nilotinib treatment.

Although bosutinib is a SRC-kinase inhibitor and SRCkinases play an important role in T- and NK-cell function, Kreutzman et al. could not find major changes in T- or NKcell phenotype or function during first-line bosutinib treatment, whereas in imatinib-treated patients, the proportion of NK-cells increased and their degranulation responses were significantly higher compared with untreated CML patients. ${ }^{29}$ The clinical value of these findings still needs to be evaluated.

\section{Bosutinib and long-term safety compared with other TKIs}

Non-hematologic AEs under bosutinib seem to occur earlier than under imatinib (e.g., liver toxicity) and at this stage, it seems as if newly occurring non-hematological toxicities behind year 1 (and even more so after year 5) are rare. ${ }^{14}$ Furthermore, no significant treatment-related irreversible toxicities have so far been described under bosutinib treatment. However, at this point, long-term follow-up data are only available for imatinib and were recently published as part of a 10-year follow-up of the IRIS trial as well as the German CML IV study whereas long-term safety data are lacking for the currently approved second-generation TKIs altogether. $^{30-33}$

Importantly, Cortes et al published a retrospective subanalysis of the vascular and cardiac safety profile of the 2 bosutinib trials. ${ }^{22}$ Particularly, on the basis of the randomized BELA study, they could show that the treatment-duration adapted rate of cardiovascular events for patients under bosutinib treatment did not exceed that of patients under imatinib treatment. Overall, increased performance status, history of vascular events, older age, and history of diabetes were prognostic for occurrence of vascular side effects.

\section{Bosutinib and concomittant medication Proton-pump inhibitors}

In a Phase I clinical trial testing bosutinib in coadministration with lansoprazole in healthy subjects, bosutinib serum levels significantly decreased after intake of the combination of both. ${ }^{34}$ Bosutinib uptake is dependent on the acid $\mathrm{pH}$-environment of the stomach and its blood levels depend on hepatic CYP3A4 metabolism, which is affected by lansoprazole. Therefore, bosutinib should not be taken together with proton-pump inhibitors. If medically indicated, shortlasting antiacids should be used and bosutinib intake should be scheduled with a sufficient time interval.

\section{Cytochrome p450 isoenzyme}

3A4Bosutinib is mostly metabolized via hepatic CYP3A4 enzymes and therefore, - as in almost all of the other CML-TKIs available, - concomitant administration of bosutinib with CYP3A4 inhibitors should be monitored carefully. Drug-drug interaction between bosutinib and certain CYP3A4 inhibitors, like aprepitant, is well understood. ${ }^{35}$ However, an algorithm, which can be used to predict drug-drug and drug-disease interactions in patients being treated with weak/moderate CYP3A4 inhibitors as well as in patients with hepatic or renal impairment has been published for bosutinib by Ono et al. ${ }^{36}$ The algorithm allows prediction of bosutinib exposures under different circumstances; however, until now, it has not been tested in a real-life setting. ${ }^{36}$

\section{Bosutinib compared with other TKIs}

As shown in Table 2, the efficacy of bosutinib is comparable with those of other TKIs. Unfortunately, no randomized clinical trial could be found comparing bosutinib with either dasatinib or nilotinib. In a matching-adjusted indirect treatment, comparison of bosutinib, dasatinib, nilotinib, and ponatinib (Iclusig ${ }^{\circledR}$, Ariad Pharmaceuticals Inc., Cambridge, MA, USA) regarding survival for second-line CP-CML by Muresan et al, a favorable HR of 1.6 with regard to PFS was described for bosutinib comparedwith nilotinib and dasatinib. ${ }^{37}$ However, such inter-trial comparisons are rather controversial, therefore have to be taken with extra caution and do, by no means, substitute for a direct comparison. Along this line, the HR comparing OS for all 3 TKIs was not statistically significant from 1 .

Table 2 compares the efficacy of 3 different secondgeneration TKI.

Characteristics of AEs profile are unique to each TKI. Figure 2 is adapted from a publication by Wolf et al and 
Table 2 Efficacy parameters of bosutinib, nilotinib and dasatinib in first- and second-line treatment

\begin{tabular}{llll}
\hline & Bosutinib & Nilotinib & Dasatinib \\
\hline MMR rate after 24 months of first-line therapy & $59^{19}$ & $7 I^{38}$ & $64^{39}$ \\
CCyR rate of second line treatment after imatinib failure/intolerance & $48^{9}$ & $41^{40}$ & $44^{41}$ \\
\hline
\end{tabular}

Abbreviations: CCyR, complete cytogenetic response; MMR, major molecular response.

\begin{tabular}{|c|c|c|c|c|c|}
\hline & Imatinib & Bosutinib & Nilotinib & Dasatinib & Ponatinib \\
\hline Cardiovascular risk factors & & & & & \\
\hline Arterial hypertension & & & & & \\
\hline Diabetes mellitus & & & & & \\
\hline Arteriosclerosis & & & & & \\
\hline Liver disease (or medical history of) & & & & & \\
\hline $\begin{array}{l}\text { Pulmonal disease (or medical history } \\
\text { of) }\end{array}$ & & & & & \\
\hline $\begin{array}{l}\text { Pulmonal-arterial hypertension (or } \\
\text { medical history of) }\end{array}$ & & & & & \\
\hline Pancreatitis (or history of) & & & & & \\
\hline $\begin{array}{l}\text { Diarrhea/chronic inflammatory bowel } \\
\text { disease }\end{array}$ & & & & & \\
\hline $\begin{array}{l}\text { Gastric ulcer (in need of proton pump } \\
\text { inhibitors) }\end{array}$ & & & & & \\
\hline recommendable & \multicolumn{5}{|c|}{ less recommendable } \\
\hline
\end{tabular}

Figure 2 Recommendations for TKI treatment with regard to comorbidity status.

Note: Adapted with permission from Wolf D, Brümmendorf TH, Isfort S. Individualisierte Therapie der CML: Bedeutung patienten- und krankheitsspezifischer Parameter für eine optimale Therapiewahl. [Individualized therapy for CML: importance of patient- and illness- specific parameters in order to choose the optimal therapy]. Thieme Praxis Report. 2015;7:9. ${ }^{42}$

shows recommendations for TKI treatment with regard to comorbidity status of each patient. ${ }^{42}$

It is advisable that the treating physician is aware of the specific side-effect profile of the administered TKI in order to inform the patient upfront, thereby making upcoming side effects less alarming and more manageable. Steegmann et al published valuable advice on how to handle AEs in all available TKIs that are applied in patients suffering from CML. ${ }^{43}$ As described previously, even more specific information exists for bosutinib. ${ }^{26}$ 


\section{Future perspectives}

At present, bosutinib is a well-established treatment alternative in the third and later lines of treatment of CML patients. Most physicians might also consider it in second-line treatment for patients with cardiovascular or respiratory diseases. The recently published positive results from the first-line BFORE trial might point to a potential use of the drug in first-line therapy in the near future.

Kota et al presented new data on an analysis on dose reductions in 2 bosutinib trials (NCT00261846 and NCT00574873) ${ }^{44}$ In this analysis, dose reduction seemed to be safe with regard to efficacy and did succeed in decreasing rates of gastrointestinal toxicity (comparison of 1 cohort taking $500 \mathrm{mg}$ /day to patients taking $400 \mathrm{mg} /$ day: diarrhea: $84 \%$ vs $50 \%$ in the second-/third-line/advanced stages trial, $70 \%$ vs $41 \%$ in the first-line trial; nausea: $45 \%$ vs $23 \%$ (secondthird-line/advanced stages trial), $34 \%$ vs $21 \%$ (first-line trial); vomiting: $33 \%$ vs $21 \%$ (second-/third-line/advanced stages trial), $28 \%$ vs $22 \%$ [first-line trial]) or to $300 \mathrm{mg} /$ day (diarrhea: $85 \%$ vs $31 \%$ [second-/third-line/advanced stages trial]), $75 \%$ vs $38 \%$ (first-line trial); nausea: $43 \%$ vs $14 \%$ (second-/ third-line/advanced stages trial), $43 \%$ vs $21 \%$ (first-line trial); and vomiting: $34 \%$ vs $11 \%$ (second-/third-line/advanced stages trial), $34 \%$ vs $18 \%$ (first-line trial). As these data have been gathered retrospectively, within the ongoing BODO trial currently recruiting in Germany (NCT03205267), a step-in dose adjustment at the start of bosutinib is being performed with the aim to reduce early onset GI toxicity. In this ongoing trial, patients start with $300 \mathrm{mg}$ and increase the dose by $100 \mathrm{mg}$ every 14 days if no AEs $\geq$ Grade 2 are occurring. The optimal daily dose providing optimal efficacy with fewest AEs possible is still missing and will be a promising challenge to find. ${ }^{45}$

As treatment-free survival is becoming a more and more relevant treatment endpoint for patients and their physicians outside clinical trials not only in first line but also in later treatment lines in CP, it is important to note that data on treatment discontinuation after bosutinib treatment is currently missing altogether. Future trials investigating whether favorable response rates seen in first- and potentially later-treatment lines will translate also into favorable rates of treatment-free remissions under bosutinib are therefore urgently needed.

\section{Disclosure}

SI received Honoraria (Advisory boards, lectures) from BMS, Novartis, Pfizer, Ariad (meanwhile Incyte) and travel grants from Roche, Mundipharma, Amgen, Hexal, Pfizer and
Novartis. THB serves on the Adisory boards of Novartis, Pfizer, Ariad (now Incyte) and provides research support for Novartis and Pfizer. The authors report no other conflicts of interest in this work.

\section{References}

1. Puttini M, Coluccia AM, Boschelli $\mathrm{F}$, et al. In vitro and in vivo activity of SKI-606, a novel Src-Abl inhibitor, against imatinib-resistant BcrAbl+ neoplastic cells. Cancer Res. 2006;66(23):11314-11322.

2. Remsing Rix LL, Rix U, Colinge J, et al. Global target profile of the kinase inhibitor bosutinib in primary chronic myeloid leukemia cells. Leukemia. 2009;23(3):477-485.

3. Golas JM, Arndt K, Etienne C, et al. SKI-606, a 4-anilino-3-quinolinecarbonitrile dual inhibitor of Src and Abl kinases, is a potent antiproliferative agent against chronic myelogenous leukemia cells in culture and causes regression of K562 xenografts in nude mice. Cancer Res. 2003;63(2):375-381.

4. Abbas R, Hsyu PH. Clinical pharmacokinetics and pharmacodynamics of bosutinib. Clin Pharmacokinet. 2016;55(10):1191-1204.

5. Levinson NM, Boxer SG. Structural and spectroscopic analysis of the kinase inhibitor bosutinib and an isomer of bosutinib binding to the $\mathrm{Abl}$ tyrosine kinase domain. PLoS One. 2012;7(4):e29828.

6. Amsberg GK, Koschmieder S. Profile of bosutinib and its clinical potential in the treatment of chronic myeloid leukemia. Onco Targets Ther. 2013;6:99-106.

7. Cortes JE, Gambacorti-Passerini C, Kim DW, et al. Effects of bosutinib treatment on renal function in patients with Philadelphia Chromosome-Positive Leukemias. Clin Lymphoma Myeloma Leuk. 2017;17(10):684-695.

8. Khoury HJ, Cortes JE, Kantarjian HM, et al. Bosutinib is active in chronic phase chronic myeloid leukemia after imatinib and dasatinib and/or nilotinib therapy failure. Blood. 2012;119(15):3403-3412.

9. Gambacorti-Passerini C, Brummendorf TH, Kim DW, et al. Bosutinib efficacy and safety in chronic phase chronic myeloid leukemia after imatinib resistance or intolerance: minimum 24-month follow-up. Am J Hematol. 2014;89(7):732-742.

10. Gambacorti-Passerini C, Kantarjian HM, Kim DW, et al. Long-term efficacy and safety of bosutinib in patients with advanced leukemia following resistance/intolerance to imatinib and other tyrosine kinase inhibitors. Am J Hematol. 2015;90(9):755-768.

11. Atilla E, Ataca P, Ozyurek E, Erden I, Gurman G. Successful bosutinib experience in an elderly acute lymphoblastic leukemia patient with suspected central nervous system involvement transformed from chronic myeloid leukemia. Case Rep Hematol. 2015;2015:689423.

12. Whiteley J, Reisman A, Shapiro M, Cortes J, Cella D. Health-related quality of life during bosutinib (SKI-606) therapy in patients with advanced chronic myeloid leukemia after imatinib failure. Curr Med Res Opin. 2016;32(8):1325-1334.

13. Cortes JE, Khoury HJ, Kantarjian HM, et al. Long-term bosutinib for chronic phase chronic myeloid leukemia after failure of imatinib plus dasatinib and/or nilotinib. Am J Hematol. 2016;91(12):1206-1214.

14. Brümmendorf TH, Gambacorti-Passerini C, Kim DW, et al. 900 Second-line bosutinib in patients with Chronic Phase Chronic Myeloid Leukemia (CP CML) Resistant or Intolerant to Prior Imatinib: an 8-year update [Abstract]. ASH Annual Meeting 2017; December 11.

15. Cortes JE, Kantarjian HM, BrummendorfTH, et al. Safety and efficacy of bosutinib (SKI-606) in chronic phase Philadelphia chromosomepositive chronic myeloid leukemia patients with resistance or intolerance to imatinib. Blood. 2011;118(17):4567-4576.

16. Brümmendorf TH, Cortes JE, Khoury HJ, et al. Factors influencing long-term efficacy and tolerability of bosutinib in chronic phase chronic myeloid leukaemia resistant or intolerant to imatinib. Br J Haematol. 2016;172(1):97-110. 
17. Trask PC, Cella D, Besson N, Kelly V, Masszi T, Kim DW. Healthrelated quality of life of bosutinib (SKI-606) in imatinib-resistant or imatinib-intolerant chronic phase chronic myeloid leukemia. Leuk Res. 2012;36(4):438-442.

18. Garcia-Gutierrez V, Martinez-Trillos A, Lopez Lorenzo JL, et al. Bosutinib shows low cross intolerance, in chronic myeloid leukemia patients treated in fourth line. Results of the Spanish compassionate use program. Am J Hematol. 2015;90(5):429-433.

19. Cortes JE, Kim DW, Kantarjian HM, Brummendorf TH, et al. Bosutinib versus imatinib in newly diagnosed chronic-phase chronic myeloid leukemia: results from the BELA trial. J Clin Oncol. 2012;30(28):3486-3492.

20. Gambacorti-Passerini C, Cortes JE, Lipton JH, et al. Safety of bosutinib versus imatinib in the phase 3 BELA trial in newly diagnosed chronic phase chronic myeloid leukemia. Am J Hematol. 2014;89(10):947-953.

21. Brummendorf TH, Cortes JE, de Souza CA, et al. Bosutinib versus imatinib in newly diagnosed chronic-phase chronic myeloid leukaemia: results from the 24-month follow-up of the BELA trial. Br J Haematol. 2015;168(1):69-81.

22. Cortes JE, Jean Khoury H, Kantarjian H, et al. Long-term evaluation of cardiac and vascular toxicity in patients with Philadelphia chromosome-positive leukemias treated with bosutinib. Am J Hematol. 2016;91(6):606-616.

23. Cortes JE, Gambacorti-Passerini C, Deininger MW, et al. Bosutinib versus imatinib for newly diagnosed chronic myeloid leukemia: results from the randomized BFORE trial. J Clin Oncol. 2017:JCO2017747162.

24. Gambacorti-Passerini C DM, Mauro MJ, Chuah C, et al. Bosutinib Vs Imatinib for Newly Diagnosed Chronic Myeloid Leukemia (CML) in the BFORE Trial: 18 Month Follow-up [Abstract]. ASH Annual Meeting 2017; December 11.

25. Gambacorti-Passerini C, Aroldi A, Cordani N, Piazza R. Chronic myeloid leukemia: second-line drugs of choice. Am J Hematol. 2016;91(1):67-75.

26. Ault PS, Rose Pharm DJ, Nodzon Ph DL, Kaled ES. Bosutinib therapy in patients with chronic myeloid leukemia: practical considerations for management of side effects. J Adv Pract Oncol. 2016;7(2):160-175.

27. Isfort S, Keller-v Amsberg G, Schafhausen P, Koschmieder S, Brummendorf TH. Bosutinib: a novel second-generation tyrosine kinase inhibitor. Recent Results Cancer Res. 2014;201:81-97.

28. Bartolovic K, Balabanov S, Hartmann U, et al. Inhibitory effect of imatinib on normal progenitor cells in vitro. Blood. 2004;103(2): 523-529.

29. Kreutzman A, Koskenvesa P, Tiina K, et al. Immunological monitoring of CML patients during first-line bosutinib and imatinib treatment. Blood. 2016;128:3069.

30. Kalmanti L, Saussele S, Lauseker M, et al. Safety and efficacy of imatinib in CML over a period of 10 years: data from the randomized CML-study IV. Leukemia. 2015;29(5):1123-1132.

31. Hochhaus A, Saglio G, Hughes TP, et al. Long-term benefits and risks of frontline nilotinib vs imatinib for chronic myeloid leukemia in chronic phase: 5-year update of the randomized ENESTnd trial. Leukemia. 2016;30(5):1044-1054.
32. Cortes JE, Jimenez CA, Mauro MJ, Geyer A, Pinilla-Ibarz J, Smith BD. Pleural effusion in dasatinib-treated patients with chronic myeloid leukemia in chronic phase: identification and management. Clin Lymphoma Myeloma Leuk. 2017;17(2):78-82.

33. Hehlmann R, Lauseker M, Saussele S, et al. Assessment of imatinib as first-line treatment of chronic myeloid leukemia: 10-year survival results of the randomized CML study IV and impact of non-CML determinants. Leukemia. 2017;31(11):2398-2406.

34. Abbas R, Leister C, Sonnichsen D. A clinical study to examine the potential effect of lansoprazole on the pharmacokinetics of bosutinib when administered concomitantly to healthy subjects. Clin Drug Investig. 2013;33(8):589-595.

35. Hsyu PH, Pignataro DS, Matschke K. Effect of aprepitant, a moderate CYP3A4 inhibitor, on bosutinib exposure in healthy subjects. Eur $J$ Clin Pharmacol. 2017;73(1):49-56.

36. Ono C, Hsyu PH, Abbas R, Loi CM, Yamazaki S. Application of physiologically based pharmacokinetic modeling to the understanding of bosutinib pharmacokinetics: prediction of drug-drug and drug-disease interactions. Drug Metab Dispos. 2017;45(4):390-398.

37. Muresan B, Mamolo C, Cappelleri JC, et al. Matching-adjusted indirect treatment comparison of bosutinib, dasatinib, nilotinib and ponatinib on survival for second line chronic phase chronic myeloid leukemia patients. Blood. 2016;128:3095

38. Kantarjian HM, Hochhaus A, Saglio G, et al. Nilotinib versus imatinib for the treatment of patients with newly diagnosed chronic phase, Philadelphia chromosome-positive, chronic myeloid leukaemia: 24-month minimum follow-up of the phase 3 randomised ENESTnd trial. Lancet Oncol. 2011;12(9):841-851.

39. Kantarjian HM, Shah NP, Cortes JE, et al. Dasatinib or imatinib in newly diagnosed chronic-phase chronic myeloid leukemia: 2-year follow-up from a randomized phase 3 trial (DASISION). Blood. 2012;119(5):1123-1129.

40. Kantarjian H, Giles FJ, Bhalla KN, et al. Nilotinib in chronic myeloid leukemia patients in chronic phase (CML-CP) with imatinib resistance or intolerance: 24-month follow-up results of a phase 2 study. Haematologica. 2009;254.

41. Kantarjian H, Pasquini R, Levy V, et al. Dasatinib or high-dose imatinib for chronic-phase chronic myeloid leukemia resistant to imatinib at a dose of 400 to 600 milligrams daily: two-year follow-up of a randomized phase 2 study (START-R). Cancer. 2009;115(18):4136-4147.

42. Wolf $\mathrm{D}$, Brümmendorf TH, Isfort $\mathrm{S}$. Individualisierte Therapie der CML: Bedeutung patienten- und krankheitsspezifischer Parameter für eine optimale Therapiewahl. [Individualized therapy for CML: importance of patient- and illness- specific parameters in order to choose the optimal therapy]. Thieme Praxis Report. 2015;7:9. German.

43. Steegmann JL, Baccarani M, Breccia M, et al. European leukemiaNet recommendations for the management and avoidance of adverse events of treatment in chronic myeloid leukaemia. Leukemia. 2016;30(8): $1648-1671$

44. Kota V, Gambacorti-Passerini C, Corts JE, et al. Efficacy and safety following bosutinib dose reduction in patients with Philadelphia chromosome-positive chronic myeloid leukemia. Blood. 2016;128:1921.

45. Kong JH, Khoury HJ, Kim AS, Hill BG, Kota V. The safety of Bosutinib for the treatment of chronic myeloid leukemia. Expert Opin Drug Saf. 2017;16(10):1203-1209.
Journal of Blood Medicine

\section{Publish your work in this journal}

The Journal of Blood Medicine is an international, peer-reviewed, open access, online journal publishing laboratory, experimental and clinical aspects of all aspect pertaining to blood based medicine including but not limited to: Transfusion Medicine; Blood collection, Donor issues, Transmittable diseases, and Blood banking logistics; Immunohematology; Artificial and alternative

\section{Dovepress}

blood based therapeutics; Hematology; Biotechnology/nanotechnology of blood related medicine; Legal aspects of blood medicine; Historical perspectives. The manuscript management system is completely online and includes a very quick and fair peer-review system. Visit http://www.dovepress.com/ testimonials.php to read real quotes from published authors. 